\title{
Design and Performance Analysis of a Multi Wavelength Terahertz Modulator Based on Triple-Lattice Photonic Crystals
}

\author{
Ke Ji, Heming Chen*, and Wen Zhou \\ Nanjing University of Posts and Telecommunications, Nanjing, Jiangsu 210023, China
}

(Received July 11, 2014 : revised September 12, 2014 : accepted September 16, 2014)

\begin{abstract}
Terahertz (THz) communication has important applications in high-speed and ultra broadband wireless access networks. The $\mathrm{THz}$ modulator is one of the key devices in a $\mathrm{THz}$ communications system. Wavelength division multiplexing (WDM) can expand the capacity of $\mathrm{THz}$ communications systems, so research on multi wavelength $\mathrm{THz}$ modulators has significant value. By combining photonic-crystal and $\mathrm{THz}$ technology, a novel type of multi wavelength $\mathrm{THz}$ modulator based on a triple-lattice photonic crystal is proposed in this paper. Compared to a compound-lattice photonic crystal, a triple-lattice photonic crystal has a larger gap width of 0.196 . Simulation results show that six beams of $\mathrm{THz}$ waves can be modulated simultaneously with high performance. This modulator's extinction ratio is as large as $34.25 \mathrm{~dB}$, its insertion loss is as low as $0.147 \mathrm{~dB}$, and its modulation rate is $2.35 \mathrm{GHz}$.
\end{abstract}

Keywords : Multi-wavelength, Photonic crystal, THz wave, Triple lattice, Modulator

OCIS codes : (230.4110) Modulators; (300.6495) Spectroscopy, terahertz; (350.4238) Nanophotonics and photonic crystals; (060.4080) Modulation; (070.7345) Wave propagation

\section{INTRODUCTION}

Nowadays the demand for broadband data communication is growing rapidly, owing to the information explosion. Optical communications systems have been researched for a long time, and the study of $\mathrm{THz}$ communications systems has become an inevitable trend. The $\mathrm{THz}$ modulator is one of the key devices in a $\mathrm{THz}$ communications system. Recently, there has been growing interest in studying $\mathrm{THz}$ wave modulators, especially multi wavelength $\mathrm{THz}$ modulators [1-3]. Currently dual-wavelength and four-wavelength $\mathrm{THz}$ modulators based on a compound-lattice photonic crystal (PC) have been proposed $[4,5]$. A novel type of six-wavelength $\mathrm{THz}$ modulator based on a triple-lattice photonic crystal is proposed in this paper.

As is well known, various methods may be used to realize $\mathrm{THz}$ wave modulation $[6,7]$. The $\mathrm{PC}$ material plays an important role in a $\mathrm{THz}$ modulator because of its excellent characteristics and production technology $[8,9]$. A PC has the characteristics of a photonic band gap (PBG) and photon localization $[10,11]$. A high-efficiency, controllable multi wavelength photonic crystal modulator can be fabricated by using a PC with defects. Using the disappearing-defect-mode theory, we have designed a six-wavelength $\mathrm{THz}$ modulator that has the advantages of high extinction ratio and low insertion loss.

\section{MODULATION MECHANISM}

Defect-mode disappearance is based on the photon-localization characteristic of a PC. This characteristic of a PC is due to the combination of both point and line defects. Line defects are introduced as waveguides to transmit $\mathrm{THz}$ wave with frequencies located in the PBG, while point defects are used as resonant cavities, in which only $\mathrm{THz}$ frequencies in accord with the resonant frequency (or defect-mode frequency) can be selected. Figure 1 is a schematic diagram of the modulation based on defect-mode disappearance. When the point defects are filled with a tunable material and the pump light is applied to the point defects, the refractive index of the material will change and the defect modes of the point defects will disappear. Therefore, the input $\mathrm{THz}$ wave cannot resonate in the cavity and the modulator is in the "off" state, as shown in Fig. 1(a). However, if there is no pump light, the $\mathrm{THz}$ wave will resonate in the cavity and the modulator

\footnotetext{
*Corresponding author: chhm@njupt.edu.cn

Color versions of one or more of the figures in this paper are available online.
} 


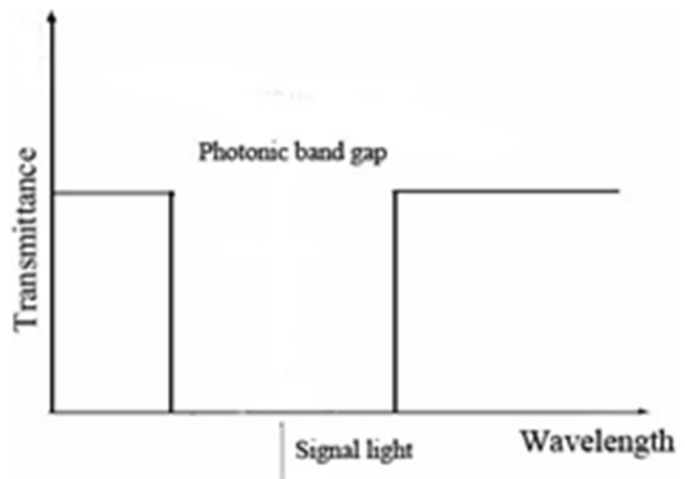

(a)

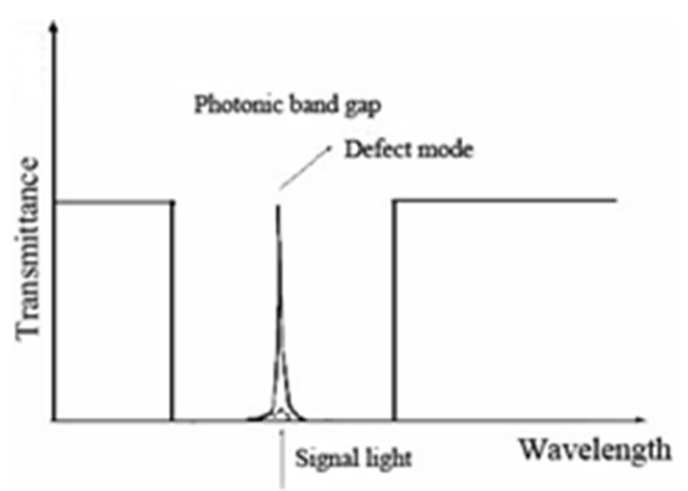

(b)

FIG. 1. Schematic diagram of modulation based on defect-mode disappearance: (a) "off” state, (b) “on" state.

is in the "on" state, as shown in Fig. 1(b) [12, 13].

\section{STRUCTURAL DESIGN AND PERFORMANCE ANALYSIS}

\subsection{Structural Design}

A triple-triangular-lattice $\mathrm{PC}$ is composed of three triangularlattice photonic crystals, including circular, square, and triangular dielectric cylinders. Figure 2 shows the lattice structure of the PC. Its structural parameters are: lattice constant $a=92 \mu \mathrm{m}$, radius of circular dielectric cylinders $r=13.9393$ $\mu \mathrm{m}$, width of square dielectric cylinders $w=25.0909 \mu \mathrm{m}$, rotation angle of square dielectric cylinders $\theta=45^{\circ}$, base length of triangular dielectric cylinders $w^{\prime}=41.8181 \mu \mathrm{m}$, and height of triangular dielectric cylinders $h=30.6666 \mu \mathrm{m}$. The material of the dielectric cylinders is silicon ( $\mathrm{Si}$ ) with refractive index $n_{1}=3.418$ in the $\mathrm{THz}$ region. The substrate material is air with refractive index $n_{2}=1$.

The band structure of this triple-triangular-lattice PC for TE waves, as calculated by the plane-wave method (PWM), is shown in Fig. 3.

The shaded part in Fig. 3 is the PBG. The normalized frequency $a / \lambda$ of the $\mathrm{PBG}$ is $0.326-0.522$, corresponding to wavelengths of $176.24-282.2 \mu \mathrm{m}$. The width of the PBG

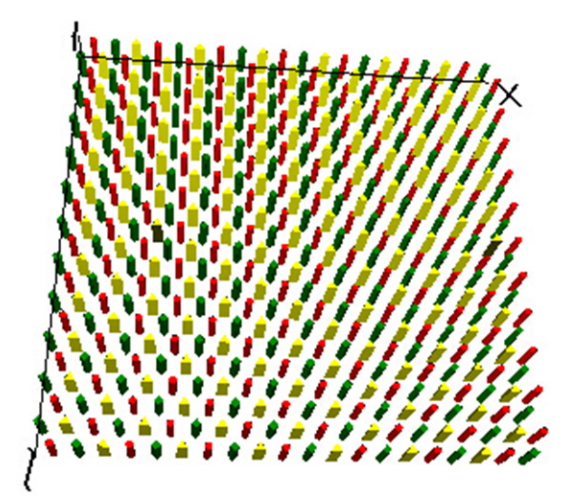

(a)

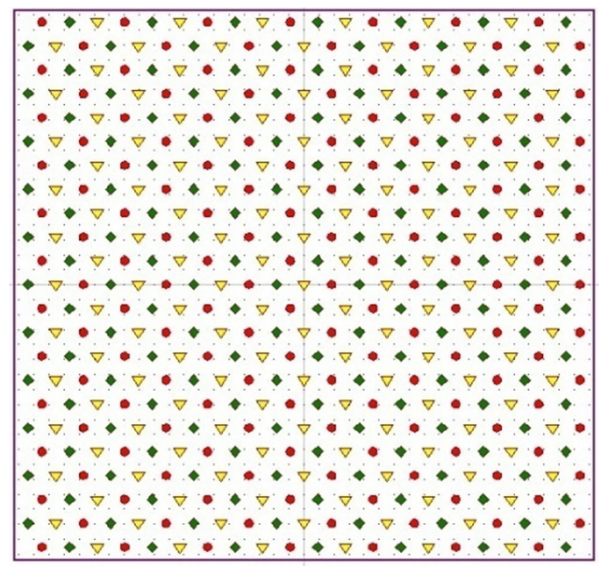

(b)

FIG. 2. The structure of the triple-triangular-lattice PC: (a) three-dimensional, (b) two-dimensional.

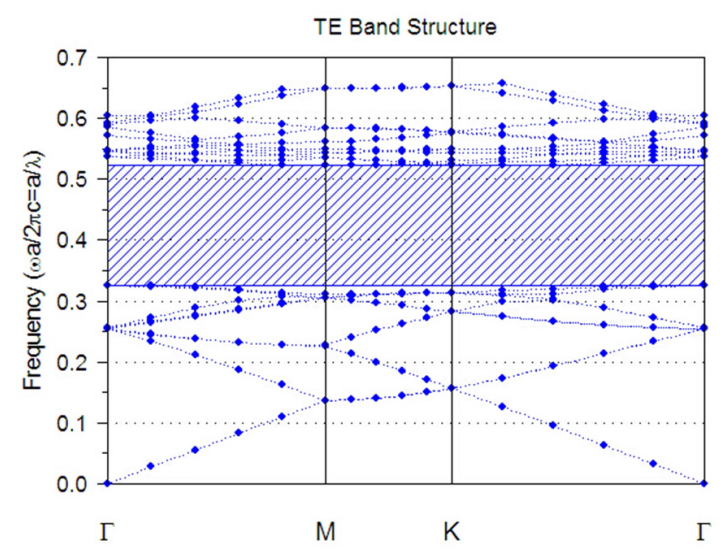

FIG. 3. The PBG diagram of the triple-triangular-lattice structure.

is 0.196 , which is much wider than that of a compoundlattice structure $[4,5]$.

To achieve multi wavelength modulation, two point defects and two line defects are introduced into the photonic crystal at the same time, as shown in Fig. 4. The point defects and line defects are directly coupled. 


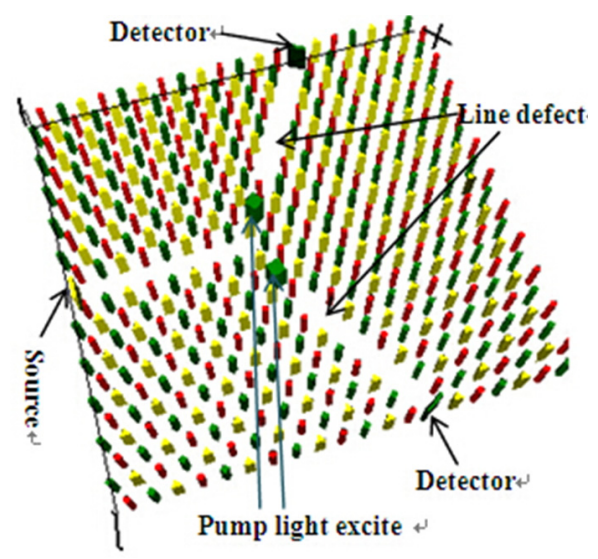

(a)

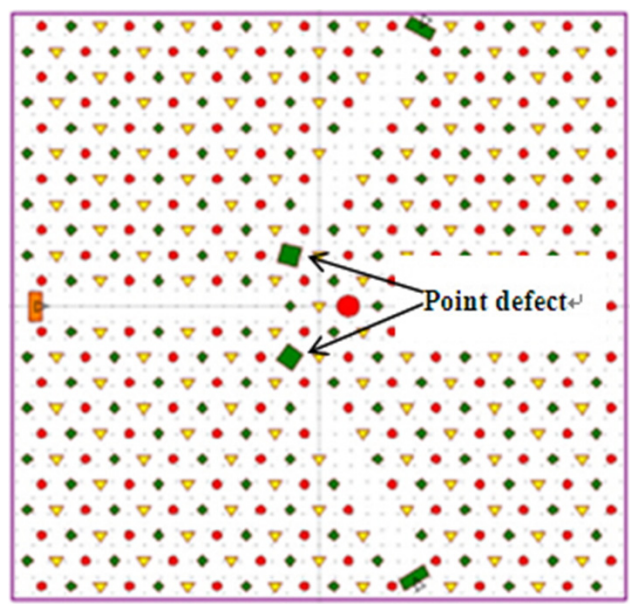

(b)

FIG. 4. The structural model of the multi wavelength photoniccrystal modulator for the $\mathrm{THz}$ range: (a) three-dimensional view, (b) two-dimensional view.

The tunable material used in the point defects is gallium arsenide (GaAs), for which the THz refractive index in the photo-excited condition is complex and defined as $N=n-i n^{\prime}$, where $n$ and $n^{\prime}$ are the real and the imaginary parts of the refractive index respectively. The complex refractive index $N$ depends on the wavelength and intensity of the external excitation light wave (pump light) [14]. According to the modulation mechanism mentioned above, when the pump (wavelength $810 \mathrm{~nm}$ ) intensity is $0.4 \mu \mathrm{J} / \mathrm{cm}^{2}, n^{\prime}$ of $\mathrm{GaAs}$ changes and the defect modes of the point defects disappear. Thus the input $\mathrm{THz}$ wave cannot resonate in the cavity and the modulator is in the "off" state. However, if the pump light is absent, the $\mathrm{THz}$ wave will resonate in the cavity and the modulator is in the "on" state.

In Fig. 4 the width of the upper square point defect is $w_{1}=2.49 w$ and the rotation angle of the upper point defect is $15^{\circ}$, while the width of the lower square point defect is $w_{1}=2.49 w$ and its rotation angle is $35^{\circ}$. The upper and lower line defects used to transmit the beams of the $\mathrm{THz}$ wave correspond to the upper and lower point defects respectively. The larger red, circular dielectric cylinder

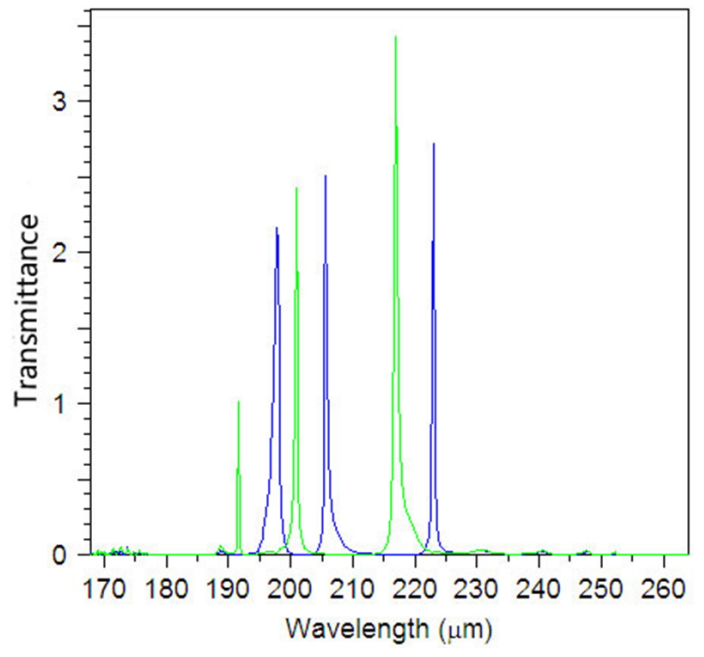

FIG. 5. The transmission spectrum of the photonic-crystal modulator.

in Fig. 4 is the result of fine tuning used to increase the transmittance of the defect modes of the square point defects.

Figure 5 displays the simulation results for the transmission spectrum. Six beams of the $\mathrm{THz}$ wave propagate through the modulator when the modulator is in "on" state, as shown. Each square point defect can modulate three beams of the $\mathrm{THz}$ wave. The wavelengths of the three defect modes produced by the upper square point (blue line) are $197.86 \mu \mathrm{m}, 205.64 \mu \mathrm{m}, 222.91 \mu \mathrm{m}$ respectively. The wavelengths of the three defect modes produced by the lower square point (green line) are $191.67 \mu \mathrm{m}, 200.92 \mu \mathrm{m}$, $216.82 \mu \mathrm{m}$ respectively. The sum of the numbers of defect modes produced by upper and lower square points is six.

\subsection{Performance Analysis}

For the case of the six beams of the $\mathrm{THz}$ wave being incident simultaneously, the extinction ratio and insertion loss of the modulator are analyzed in detail below. From Fig. 5, the six incident wavelengths are set as $191.67 \mu \mathrm{m}$, $197.86 \mu \mathrm{m}, 200.92 \mu \mathrm{m}, 205.64 \mu \mathrm{m}, 216.82 \mu \mathrm{m}$, and 222.91 $\mu \mathrm{m}$ respectively. All of the incident $\mathrm{THz}$ waves are Gaussian and continuous.

The extinction ratio is an important index to evaluate the capacity of modulation. The time-domain steady-state response is shown in Fig. 6. As registered by the upper detector in Fig. 4, Figs. 6(a) and 6(b) show the "on" and "off" states of the modulator respectively. As registered by the lower detector in Fig. 4, Figs. 6(c) and 6(d) show the "on" and "off" states of the modulator respectively.

The definition of extinction ratio is

$$
\eta=10 \log \left(I_{\max } / I_{\min }\right)
$$

where $I_{\max }$ is the maximum transmission intensity of the 


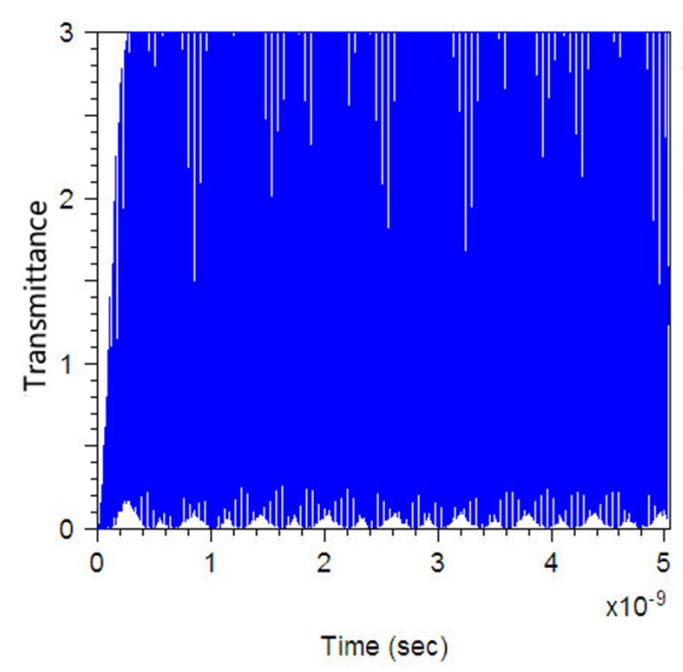

(a)

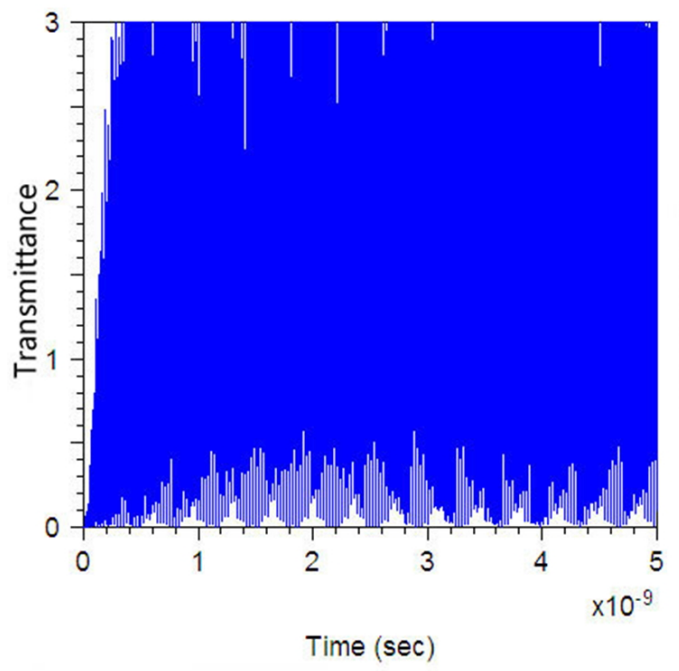

(c)



(b)

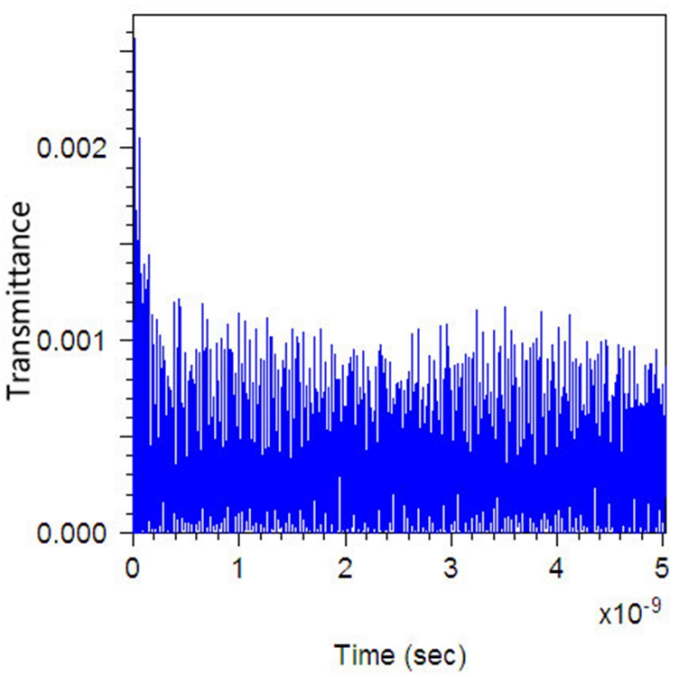

(d)

FIG. 6. Time-domain steady-state response of (a) "on" state and (b) "off" state as registered by the upper detector, and of (c) "on" state and (d) "off" state as registered by the lower detector.

THz wave after modulation, $I_{\min }$ is the minimum transmission intensity of the $\mathrm{THz}$ wave after modulation, and $\eta$ is the extinction ratio of the modulator. The value of $I_{\max }$ is the sum of the values in Figs. 6(a) and 6(c), while the value of $I_{\min }$ is the sum of the values in Figs. 6(b) and 6(d) respectively. From Fig. 6 we can obtain the value of $I_{\max }$ as 5.8 and the value of $I_{\min }$ as $2.179 \times 10^{-3}$. From Eq (1), we obtain an extinction ratio of $34.25 \mathrm{~dB}$.

The definition of insertion loss is

$$
\gamma=10 \log \left(I_{\text {in }} / I_{\max }\right)
$$

where $I_{\text {in }}$ is the intensity of the incident $\mathrm{THz}$ wave and $I_{\max }$ is the maximum transmission intensity of the $\mathrm{THz}$ wave after modulation. The value of $I_{\text {in }}$ is 6 , and from $\mathrm{Eq}$ (2) we obtain an insertion loss of $0.147 \mathrm{~dB}$.
The definition of the modulation rate is

$$
\begin{aligned}
& v=1 / T \\
& \mathrm{~T}=T_{1}+T_{2}
\end{aligned}
$$

where $T$ is the response time of the system and $v$ is the modulation rate. $T$ is determined by the sum of the response time of $G_{a} A_{s}$ material $E$ 's change in refractive index $\left(T_{1}\right)$ and the stability time of the "on" or "off" state of the modulator $\left(T_{2}\right)$. Here $T_{1}$ is $100 \mathrm{ps}$ [15] and $T_{2} 3.2499$ $\times 10-{ }^{10} s$ from Fig. 6 . The modulation rate is $2.35 \mathrm{GHz}$, as obtained from Eqs. (3) and (4).

The steady-state $\mathrm{THz}$ wave field intensity distributions of in "on" $\left(n^{\prime}=0\right)$ and "off" states $\left(n^{\prime}=2.55\right)$ are shown in Figs. 7(a) and 7(b) respectively.

Figure 7 shows that the six beams of the $\mathrm{THz}$ wave 


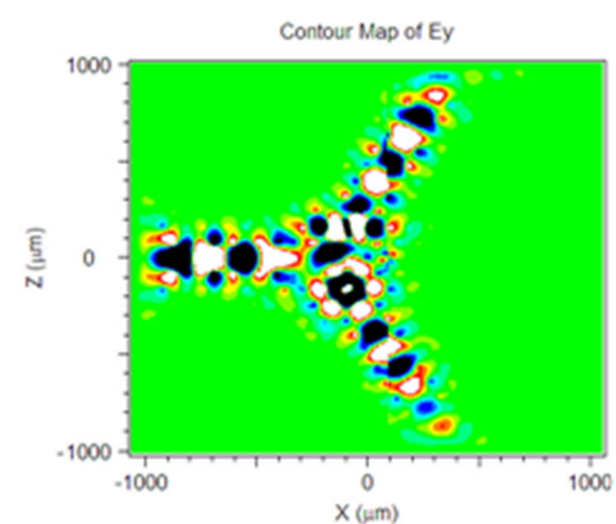

(a)

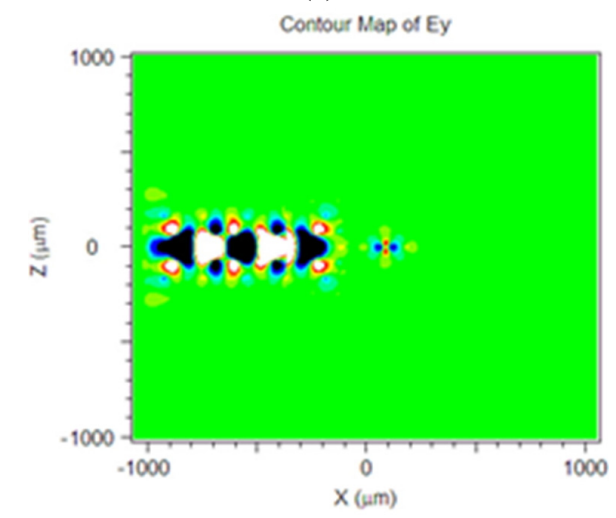

(b)

FIG. 7. Steady-state THz wave field intensity distribution of $E_{y}$ : (a) in the "on" state $\left(n^{\prime}=0\right)$, (b) in the "off" state $\left(n^{\prime}=2.55\right)$.

were modulated effectively by the multi-wavelength photoniccrystal $\mathrm{THz}$ modulator with a large extinction ratio.

The simulation results show that this novel multi wavelength photonic-crystal $\mathrm{THz}$ modulator can effectively modulate a $\mathrm{THz}$ wave, with large extinction ratio and low insertion loss.

\section{CONCLUSION}

A novel multi wavelength $\mathrm{THz}$ modulator based on a triple-triangle-lattice-structure photonic crystal is proposed. By changing the GaAs refractive index, the modulation of six wavelengths $(191.67 \mu \mathrm{m}, 197.86 \mu \mathrm{m}, 200.92 \mu \mathrm{m}$, $205.64 \mu \mathrm{m}, 216.82 \mu \mathrm{m}$, and $222.91 \mu \mathrm{m})$ can be realized. The extinction ratio is as large as $34.25 \mathrm{~dB}$, the insertion loss as low as $0.147 \mathrm{~dB}$, and the modulation rate $2.35 \mathrm{GHz}$. This modulator presents a theoretical foundation for research in communications devices for the $\mathrm{THz}$ range, and can also meet the requirements of future $\mathrm{THz}$ wave communications systems better.

\section{ACKNOWLEDGMENT}

This work was supported by the National Natural Science Foundation of China (project No. 61077084).

\section{REFRENCES}

1. H. M. Chen, J. Su, J. L. Wang, and X. Y. Zhao, "Opticallycontrolled high-speed terahertz wave modulator based on nonlinear photonic crystals," Opt. Express 19, 3599-3603 (2011).

2. L. Fekete, F. Kadlec, H. Nemec, and P. Kuzel, "Fast one-dimensional photonic crystal modulators for the terahertz range," Opt. Express 15, 8898-8912 (2007).

3. H. C. Nguyen, Y. Sakai, M. Shinkawa, N. Ishikura, and T. Baba, " $10 \mathrm{~Gb} / \mathrm{s}$ operation of photonic crystal silicon optical modulator," Opt. Express 19, 13000-13007 (2011).

4. T. D. J. Li, and H. M. Chen, "Terahertz wave tunable resonator based on compound lattice photonic crystal," in Proc. The 9th International Conference on Optical Communication and Networks (ICOCN, China, Oct. 2010), pp. 402-405.

5. W. Zhou and H. M. Chen, "Study on the characteristics of quadruple-wavelength $\mathrm{THz}$ modulator based on compound lattice photonic crystal with direct-coupled structure," in Proc. The 2013 International Conference on Communication Technology (ICCT, Singapore, 2013), pp. 821-830.

6. M. Rahm, J. S. Li, and W. J. Padilla, "THz wave modulators: A brief review on different modulation techniques," J. Infrared Milli. Terahz. Waves 34, 1-27 (2013).

7. A. Figotin, Y. A. Godin, and I. Vitebsky, "Two-dimensional tunable photonic crystals," Phys. Rev. B 57, 2841-2848 (1998).

8. J. S. Li and S. Zouhdi, "Ultrafast and low-power terahertz wave modulator based on organic photonic crystal," Opt. Commun. 285, 953-956 (2012).

9. J. S. Li, "Terahertz modulator using photonic crystals," Opt. Commun. 269, 98-101 (2007).

10. E. Yablonovitch, "Inhibited spontaneous emission in solidstate physics and electronics," Phys. Rev. Lett. 58, 2059 (1987).

11. S. John, "Strong localization of photons in certain disordered dielectric super lattices," Phys. Rev. Lett. 58, 2486 (1987).

12. M. Notomi, A. Shinya, S. Mitsugi, G. Kira, E. Kuramochi, and T. Tanabe, "Optical bistable switching action of $\mathrm{Si}$ high-Q photonic-crystal nanocavities," Opt. Express 13, 2678-2687 (2005).

13. T. Chen, P. Liu, J. Liu, and Z. Hong, "A terahertz photonic crystal cavity with high Q-factors," Appl. Phys. B 115, 105-109 (2014).

14. H. F. Tiedje, H. K. Haugen, and J. S. Preston, "Measurement of nonlinear absorption coefficients in $G_{a} A_{s}$. $I_{n} P$, and $S_{i}$ by an optical pump THz probe technique," Opt. Commun. 274, 187-197 (2007).

15. L. Fekete, F. Kadlec, and P. Kuzel, "Ultrafast opto-terahertz photonic crystal modulator," Opt. Lett. 32, 680-682 (2007). 